\title{
Preface: Multivariate algorithms and information-based complexity
}

The authors of this book include several of the invited speakers in the workshop Multivariate Algorithms and Information-Based Complexity, which was part of the RICAM Special Semester on Multivariate Algorithms and their Foundations in Number Theory in the fall of 2018. The special semester consisted of four larger and two smaller workshops on various topics ranging from Pseudo-Randomness and Discrepancy Theory to Information-Based Complexity and Uncertainty Quantification. This book arises from the second workshop, which took place at the Johann Radon Institute for Computational and Applied Mathematics (RICAM) of the Austrian Academy of Sciences in Linz, Austria, on November 5-9, 2018.

Multivariate continuous problems occur in a multitude of practical applications, such as physics, finance, computer graphics, and chemistry. The number of variables involved, $d$, can be in the hundreds or thousands. The information complexity of a given problem is the minimal number of information operations required by the best algorithm to solve the problem for a prescribed set of inputs within a certain error threshold, $\varepsilon$. Typical examples of information operations are function values and linear functionals. The field of information-based complexity (IBC), founded by Traub and Wozniakowski in the 1980s, analyzes the information complexity for multivariate problems and determines how it depends on $d$ and $\varepsilon$. A crucial question is under which circumstances one can avoid a curse of dimensionality, namely, exponential growth of the information complexity with $d$. This book addresses the analysis of multivariate (continuous) problems, especially from the IBC viewpoint.

The problems discussed by the authors reflect the breadth of current inquiry under the umbrella of multivariate algorithms and IBC. The chapter entitled"The control variate integration algorithm for multivariate functions defined at scattered data points" studies a method of approximating the integral of a multivariate function, in which one uses the exact integral of a control variate based on a least-squares multivariate quasiinterpolant. Numerical examples demonstrate that such an algorithm can overcome the curse of dimensionality for multivariate least-squares fits. The second chapter, titled "An adaptive random bit multilevel algorithm for SDEs", considers the approximations of expectations for functionals applied to the solutions of stochastic differential equations by employing Monte Carlo methods based on random bits instead of random numbers. An adaptive random bit multilevel algorithm is provided and compared numerically to other methods. The chapter "RBF-based penalized least-squares approximation of noisy scattered data on the sphere" deals with the approximation of noisy scattered data on the 2-dimensional unit sphere. In particular, global and local penalized least-squares approximation based on radial basis functions (RBFs) are explored. The authors of the fourth chapter in this book, "On the power of random information", consider a problem from the core of IBC theory, 
namely the quality of random information in approximation and integration problems. Random information is compared to optimal information for such problems. It is shown that in some cases random information can be almost optimal, whereas in other cases it may yield much worse results than optimal information. The chapter "Optimality criteria for probabilistic numerical methods" lies in the field of Bayesian decision theory. To be more precise, the authors study an optimality criterion from Bayesian experimental design and consider its implied optimal information in the numerical context. Furthermore, this chapter compares this information to information commonly used in average-case-optimal numerical methods. The sixth chapter of the book, " $\varepsilon$-Superposition and truncation dimensions, and multivariate decomposition method for $\infty$-variate linear problems" deals with linear problems on weighted normed function spaces, the elements of which depend on infinitely many variables. The author focuses on the $\varepsilon$-truncation and $\varepsilon$-superposition dimension, as well as multivariate decomposition methods, which are means to reduce the number of variables in the problem to a relatively small finite number. The anchored and other types of decompositions are used. The chapter "Adaptive approximation for multivariate linear problems with inputs lying in a cone" completes the book and considers adaptive approximation algorithms for functions lying in particular subsets of certain function spaces. In contrast to much of the IBC literature, it is not assumed that the functions to be approximated lie in a ball of a certain radius, but instead in a cone. It is shown that for such problems it is an advantage to use adaptive rather than nonadaptive algorithms for function approximation.

All chapters were reviewed by renowoned experts. We wish to thank these anonymous referees for their precious help.

We would like to express our gratitude towards all speakers of the workshop for giving excellent talks on their respective fields of expertise and for contributing to the success of the workshop:

- Paul Constantine (University of Colorado),

- Martin Ehler (University of Vienna),

- Mario Hefter (TU Kaiserslautern),

- Stefan Heinrich (TU Kaiserslautern),

- Kerstin Hesse (Paderborn University),

- Fred J. Hickernell (Illinois Institute of Technology),

- James (Mac) Hyman (Tulane University),

- Thomas Müller-Gronbach (University of Passau),

- Erich Novak (University of Jena),

- Chris Oates (Newcastle University and Alan Turing Institute),

- Houman Owhadi (California Institute of Technology),

- Sergei Pereverzyev (RICAM), Leszek Plaskota (University of Warsaw),

- Paweł Przybyłowicz (AGH University of Science and Technology Kraków),

- Klaus Ritter (TU Kaiserslautern),

- Ingo Steinwart (University of Stuttgart), 
- Michaela Szölgyenyi (University of Klagenfurt),

- Greg W. Wasilkowski (University of Kentucky),

- Henryk Woźniakowski (Columbia University and University of Warsaw),

- Henry Wynn (London School of Economics),

- Larisa Yaroslavtseva (University of Passau).

We also would like to thank all those who participated in the workshop. Annette Weihs and Wolfgang Forsthuber provided valuable administrative and technical support. Further information on the RICAM Special Semester "Multivariate Algorithms and their Foundations in Number Theory” can be found at

https://www.ricam.oeaw.ac.at/specsem/specsem2018/

The financial support of the Johann Radon Institute for Computational and Applied Mathematics (RICAM) of the Austrian Academy of Sciences is gratefully acknowledged.

Fred J. Hickernell and Peter Kritzer, Chicago and Linz, March 2020. 
